\title{
Enhanced genome assembly and a new official gene set for Tribolium castaneum
}

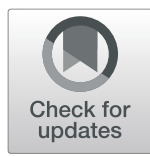

\begin{abstract}
Nicolae Herndon ${ }^{1 \dagger}$, Jennifer Shelton ${ }^{2 \dagger}$, Lizzy Gerischer ${ }^{3 \dagger}$, Panos loannidis ${ }^{4}$, Maria Ninova ${ }^{5}$, Jürgen Dönitz ${ }^{6}$, Robert M. Waterhouse ${ }^{7}$ Chun Liang $^{8}$, Carsten Damm ${ }^{9}$, Janna Siemanowski ${ }^{6}$, Peter Kitzmannn ${ }^{6}$, Julia Ulrich ${ }^{6}$, Stefan Dippel ${ }^{10}$, Georg Oberhofer ${ }^{6}$, Yonggang Hu${ }^{6}$, Jonas Schwirz ${ }^{6}$, Magdalena Schacht ${ }^{6}$, Sabrina Lehmann ${ }^{6}$, Alice Montino ${ }^{6}$, Nico Posnien ${ }^{11}$, Daniela Gurska ${ }^{12}$, Thorsten Horn ${ }^{12}$, Jan Seibert ${ }^{12}$, Iris M. Vargas Jentzsch ${ }^{12}$, Kristen A. Panfilio ${ }^{13}$, Jianwei $\mathrm{Li}^{14}$, Ernst A. Wimmer ${ }^{15}$, Dominik Stappert ${ }^{16}$, Siegfried Roth ${ }^{16}$, Reinhard Schröder ${ }^{17}$, Yoonseong Park ${ }^{18}$, Michael Schoppmeier ${ }^{19}$, Ho-Ryun Chung ${ }^{20}$, Martin Klingler ${ }^{21}$, Sebastian Kittelmann ${ }^{22}$, Markus Friedrich ${ }^{23}$, Rui Chen ${ }^{24}$, Boran Altincicek ${ }^{25}$, Andreas Vilcinskas ${ }^{26}$, Evgeny Zdobnov ${ }^{4}$, Sam Griffiths-Jones ${ }^{5}$, Matthew Ronshaugen ${ }^{5}$, Mario Stanke ${ }^{3^{*}}$, Sue J. Brown ${ }^{2^{*}}$ and Gregor Bucher ${ }^{27^{*}}$ (i)
\end{abstract}

\begin{abstract}
Background: The red flour beetle Tribolium castaneum has emerged as an important model organism for the study of gene function in development and physiology, for ecological and evolutionary genomics, for pest control and a plethora of other topics. RNA interference (RNAi), transgenesis and genome editing are well established and the resources for genome-wide RNAi screening have become available in this model. All these techniques depend on a high quality genome assembly and precise gene models. However, the first version of the genome assembly was generated by Sanger sequencing, and with a small set of RNA sequence data limiting annotation quality.

Results: Here, we present an improved genome assembly (Tcas5.2) and an enhanced genome annotation resulting in a new official gene set (OGS3) for Tribolium castaneum, which significantly increase the quality of the genomic resources. By adding large-distance jumping library DNA sequencing to join scaffolds and fill small gaps, the gaps in the genome assembly were reduced and the N50 increased to $4753 \mathrm{kbp}$. The precision of the gene models was enhanced by the use of a large body of RNA-Seq reads of different life history stages and tissue types, leading to the discovery of 1452 novel gene sequences. We also added new features such as alternative splicing, well defined UTRs and microRNA target predictions. For quality control, 399 gene models were evaluated by manual inspection. The current gene set was submitted to Genbank and accepted as a RefSeq genome by NCBI.

Conclusions: The new genome assembly (Tcas5.2) and the official gene set (OGS3) provide enhanced genomic resources for genetic work in Tribolium castaneum. The much improved information on transcription start sites supports transgenic and gene editing approaches. Further, novel types of information such as splice variants and microRNA target genes open additional possibilities for analysis.
\end{abstract}

Keywords: Tribolium castaneum, Genome, Genome assembly Tcas5.2, Reannotation, Gene prediction, Gene set OGS3, RefSeq genome, Gene annotation, microRNA, miRNA

\footnotetext{
* Correspondence: mario.stanke@uni-greifswald.de; sjbrown@ksu.edu; gbucher1@uni-goettingen.de

${ }^{\dagger}$ Nicolae Herndon, Jennifer Shelton and Lizzy Gerischer contributed equally

to this work.

${ }^{3}$ Institut für Mathematik und Informatik, Universität Greifswald, Greifswald,

Germany

${ }^{2}$ Division of Biology, Kansas State University, Manhattan, KS 66506, USA

${ }^{27}$ Georg-August-Universität Göttingen, Göttingen, Germany

Full list of author information is available at the end of the article
}

(c) The Author(s). 2020 Open Access This article is distributed under the terms of the Creative Commons Attribution 4.0 International License (http://creativecommons.org/licenses/by/4.0/), which permits unrestricted use, distribution, and

reproduction in any medium, provided you give appropriate credit to the original author(s) and the source, provide a link to the Creative Commons license, and indicate if changes were made. The Creative Commons Public Domain Dedication waiver (http://creativecommons.org/publicdomain/zero/1.0/) applies to the data made available in this article, unless otherwise stated. 


\section{Background}

The red flour beetle Tribolium castaneum is an excellent insect model system for functional genetics. In many respects the biology of Tribolium is more representative of insects than that of the fly Drosophila melanogaster [1-3]. This is especially true with respect to embryonic development: The Tribolium embryo is enveloped by extraembryonic membranes like most insects [4], develops embryonic legs, displays an everted head [5] and its posterior segments are formed sequentially from a posterior segment addition zone $[6,7]$. With respect to postembryonic development, the Tribolium larval epidermal cells build most of the adult epidermis while in Drosophila they are replaced by imaginal cells [8]. In the telotrophic ovary type of Tribolium the biology of somatic stem cells can be studied independent of germline stem cells, which cease to divide prior to hatching [9]. Tribolium is also studied with respect to beetle specific evolutionary novelties such as elytra [10] and gin traps [11]. It is also amenable to studies of physiology such as the formation of the extremely hard cuticle [12], and the function of the cryptonephridial system [13], which is a model for unique adaptation to dry habitats. Odoriferous glands are studied to understand the production of toxic secretions without harming the animal [14]. Finally, Tribolium is a representative of the Coleoptera, which is the most species-rich taxon on earth [15] including many economically important pests such as leaf and snout beetles. Hence, it has been used as a model for pest control [16, 17]. In summary, Tribolium is useful for evolutionary comparisons of gene function among insects, for studying processes that are not represented in Drosophila and for pest control studies.

Research on gene function in Tribolium is fostered by an extensive toolkit. Transposon-mediated transgenesis has led to the development of imaging and misexpression tools, and has facilitated a large-scale insertional mutagenesis screen [18-24]. However, the main strength of the model system lies in its reverse genetics via RNAi. First, the RNAi response is very strong, reaching the null phenotype in those cases where a genetic mutant was available for comparison [25-28]. In addition, RNAi is environmental, i.e. cells very efficiently take up dsRNA from the hemolymph and the RNAi effect is transmitted from injected mothers to their offspring [29-31]. Based on this strength, a genome wide RNAi screen was performed (iBeetle screen), in which embryonic and other phenotypes were documented and made available via the iBeetle-Base [32-34]. Importantly, the genome wide collection of templates generated by iBeetle can be used for future screens directed at other processes. Recently, CRISPR/Cas9 mediated genome editing has been shown to work efficiently $[35,36]$.
An essential requirement for studying gene function is a high quality genome assembly and a well annotated gene set. Indeed, the first genome assembly, published in 2008 community database [37, 38] contributed significantly to the growth of the community and increased the diversity of research topics studied in Tribolium. However, in the first published Tribolium genome assembly a substantial number of scaffolds had not been anchored to any Linkage Group. Further, the first gene annotations were mainly based on the detection of sequence features by bioinformatics tools and homology to Drosophila genes and very few gene predictions were supported by RNA data. Hence, precision in the coding regions was limited, non-coding UTR sequences and transcription start sites were usually not defined and splice variants were not predicted.

Here, we made use of new sequencing and mapping techniques in order to significantly enhance the genomic resources of Tribolium. In the new Tribolium assembly, Tcas5.2, scaffold length has been increased fivefold (scaffold N50: 4753kbp). With the inclusion of RNA-Seq data, the precision of gene models was improved and additional features such as UTRs and alternative splice variants were added to 1335 gene models. 1452 newly predicted genes replaced a similar number of short genes that had been falsely predicted. The current set of gene models (OGS3) is the first NCBI RefSeq annotation for Tribolium castaneum. Based on the enhanced annotation we compared the degree of conservation of protein sequences between a number of model systems revealing Tribolium sequences appear less diverged compared to other Ecdysozoa. Moreover, with the identification of UTRs, we were able to map, for the first time in a beetle, potential target genes of the microRNA complement and identified a conserved target gene set for a conserved microRNA.

\section{Results}

Improving the scaffolding of the Tcas genome assembly

The first published Tribolium genome sequence (NCBI Tcas3.0) was based on a Sanger 7x draft assembly [38] totaling $160 \mathrm{Mb}, 90 \%$ of which was anchored to pseudomolecules or Linkage Groups (LGs) representing linkage groups in the molecular recombination map [39]. However, several large scaffolds (up to $1.17 \mathrm{Mb}$ ) were not included. To improve this draft assembly, we sequenced the paired ends of three large-insert jumping libraries (appr. $3200 \mathrm{bp}, 6800 \mathrm{bp}$, and 34,800 bp inserts, respectively). These sequences were used to link scaffolds in the Sanger assembly and fill small gaps. Further, whole genome physical maps produced from images of ultra-long individual molecules of Tribolium DNA labeled at restriction sites (BioNano Genomics) were used to validate the assembly and merge scaffolds. The entire workflow and key steps are described below. 
Using the long-insert jumping libraries, Atlas-Link (Baylor College of Medicine; www.hgsc.bcm.edu/software/atlas-link) joined neighboring anchored scaffolds and added several unplaced scaffolds, reducing the total number of scaffolds from 2320 to 2236 . Of these, three were manually split because the joined scaffolds were known to be on different linkage groups based on the molecular genetic recombination map, leading to a total of 2240 scaffolds. This analysis added formerly unplaced scaffolds to all LGs except LG4. In addition, 16 unplaced scaffolds were linked together.

We also took advantage of the new Illumina sequence information gained from the long insert jumping libraries to fill small gaps and extend contigs. GapFiller [40] added 77,556 nucleotides and closed 2232 gaps (Table 1). Specifically, the number of gaps of assigned length 50 , which actually included gaps less than 50 nucleotides long or potentially overlapping contigs, was reduced by $65.6 \%$ (from 1793 to 615).

Finally, BioNano Genomics consensus maps were used to validate and further improve the assembly (for details, see [41]). More than $81 \%$ of Tcas 5.2 was directly validated by alignment with BioNano Genomics Consensus maps, the number of scaffolds was reduced by $4 \%$ to 2148 , and the N50 increased 3-fold to $4753.0 \mathrm{~kb}$. In total, the N50 was increased almost 5-fold where superscaffolding with BioNano Genomics optical maps improved the contiguity of the assembly the most. Table 2 shows the extent to which each step of the workflow impacted the quality of the genome assembly.

\section{Re-annotation of the Tribolium genome assembly}

Re-annotation was performed using the gene finder AUGUSTUS [42]. For the current release, new data were available and incorporated as extrinsic evidence including RNA-Seq, ESTs (Expressed Sequence Tags) and protein sequences. The most impactful new information was the extensive RNA-Seq data (approximately 6.66 billion reads) covering different life stages and tissues. This allowed us to determine UTRs and alternative splice variants, which were not annotated in the previous official gene set. This increased both transcript coverage (Table 3) and the accuracy of the predicted gene features. The parameters of automated annotation were adjusted based on manual quality control of more than 500 annotations of previously published genes. The new gene set, OGS3, consists of 16,593 genes with a total of 18,536 transcripts. 15,258 (92\%) genes have one isoform, 944 (5.7\%) genes have two, 270 (1.6\%) have three and 121 $(0.7 \%)$ genes have more than three isoforms. During the re-annotation of the Tribolium gene set a basic parameter set for AUGUSTUS was developed and is now delivered with AUGUSTUS as parameter set "tribolium2012" (link for download: see Materials and Methods).

\section{Major changes in the OGS3}

We compared the previous official gene set OGS2 [37], which was 'lifted' to the new assembly, Tcas5.2, with the new OGS3 and found that 9294 genes have identical protein sequences, while 3039 genes have almost identical protein sequences (95\% minimum identity and $95 \%$ minimum coverage). 1452 genes were completely new, meaning that they did not overlap any lifted OGS2 gene above the given thresholds. A similar number (1420) of predicted genes from OGS2 do not exist anymore in OGS3. We further analyzed the "lost" and "new" genes and found that our procedure was efficient in removing false positive annotations and in detecting novel true genes. First, based on the lack of a BLAST hit in invertebrates (e-value cutoff: e-05), GO annotation or RNA-Seq

Table 1 Ungapped length and spanned gaps before and after running GapFiller

\begin{tabular}{|c|c|c|c|c|}
\hline Molecule & Ungapped length before & Spanned gaps before & Ungapped length after & Spanned gaps after \\
\hline $\mathrm{LG} 1=\mathrm{X}$ & $7,071,107$ & 301 & $7,096,881$ & 201 \\
\hline LG2 & $14,229,660$ & 359 & $14,306,202$ & 192 \\
\hline LG3 & $28,072,007$ & 1451 & $28,315,770$ & 929 \\
\hline LG4 & $11,540,046$ & 300 & $11,632,658$ & 160 \\
\hline LG5 & $14,111,830$ & 358 & $14,196,565$ & 193 \\
\hline LG6 & $8,262,430$ & 555 & $8,332,882$ & 407 \\
\hline LG7 & $15,084,119$ & 429 & $15,185,902$ & 258 \\
\hline LG8 & $12,870,760$ & 577 & $12,987,347$ & 378 \\
\hline LG9 & $14,900,846$ & 634 & $15,007,071$ & 384 \\
\hline LG10 & $7,070,154$ & 498 & $7,128,489$ & 365 \\
\hline Unplaced multi-contig & $14,079,574$ & 1111 & $14,205,681$ & 874 \\
\hline Unplaced single-contig & $4,020,722$ & - & $4,021,060$ & - \\
\hline Total & $151,313,255$ & 6573 & $152,416,508$ & 4341 \\
\hline
\end{tabular}


Table 2 Assembly improvement

\begin{tabular}{llll}
\hline Assembly & Length & Scaffolds & Scaffold N50 (kbp) \\
\hline Tcas 3.0 & $160,445,652$ & 2320 & 976.4 \\
After Atlas-Link & $160,667,144$ & 2240 & 1175.4 \\
After GapFiller & $160,744,700$ & 2240 & 1176.7 \\
After BioNano Genomics / & $165,921,904$ & 2148 & 4753.0 \\
Tcas 5.2 & & & \\
\hline
\end{tabular}

coverage we assume that the "lost" OGS2 annotations had been falsely annotated. Second, when examining the newly found genes, we observe that 528 of 1452 (36\%) genes had significant BLAST hits in other insect species. Further, 690 of 997 (69.2\%) of the new genes have at least one intron supported by RNA-Seq. New single exon genes have an average read coverage of about 550,000 reads per gene with minimum coverage of 11 reads per gene. The percentage of missing BUSCO genes was reduced from 0.7 to $0.4 \%$. Together, these metrics indicate that real genes were newly annotated. Table 4 compares important characteristics between the previous and the current OGS.

We further examined gene structure changes (not including the identification of splice variants). For this, we counted both, gene join and split events that occurred in the new gene set. Joins are indicated when the CDS of an OGS3 gene overlaped the CDSs of two or more genes from the previous gene set on the same strand. In total, we observe 949 such join events. In 485 (51\%) of these events, the new intron of an OGS3 gene was supported by spliced read alignments spanning the gap between two neighboring OGS2 genes, suggesting that the annotations had erroneously been split in the previous annotation. We detected gene split events by counting gene join events where an old OGS2 gene joined multiple OGS3 genes. We observed 424 such events. In 45 cases (10\%) the joining OGS2 intron had RNA-Seq support. Taken together, while $>50 \%$ of the joined genes were supported by sequencing data only $10 \%$ of the split events turned out to be likely false positives.

Table 3 Read alignments to OGS2 and OGS3 transcript sets. The numbers of alignments are shown. Only the best alignment(s) for each read are reported. The last row suggests that OGS2 may have a slight bias towards highly expressed genes

\begin{tabular}{lll}
\hline & OGS2 & OGS3 \\
\hline Total number of alignments & $4,634,356,882$ & $7,418,675,525$ \\
Number of alignments per transcript & 278,926 & 400,317 \\
Number of aligned reads per exon position & 285.77 & 260.45 \\
\hline
\end{tabular}

This indicated that the parameter set was adequate to enrich for true annotations in the new gene set.

\section{RNA-Seq support for the gene sets}

Analysis of differential gene expression has become an essential tool in studying the genetic basis of biological processes. Such analyses profit from a better gene model where a higher number of reads can be mapped. To test whether the new gene set performed better in such analyses, we mapped our collection of RNA-Seq reads to both (Table 3). In this analysis 6.66 billion RNA-Seq reads from Tribolium where mapped against the two gene sets (transcriptome) OGS3 and, for comparison, OGS2 with the alignment tool BLAT [43]. Alignments with less than $90 \%$ identity were discarded and only the best alignment was kept for each read. About 70\% of the reads mapped to OGS2 whereas $81 \%$ mapped to OGS3.

To evaluate the splice sites in the new gene set we compiled a set of splices suggested by gaps in RNA-Seq read alignments compared to the genomic sequence (intron candidates). These RNA-Seq read alignments where filtered by a range of criteria (see Methods). In total this set contained 65,274 intron candidates. We refer to the term multiplicity of an intron candidate as the number of reads that were found to cross a given exon-exon boundary at the identical position. Some candidate introns are likely not introns of coding genes, e.g. from alignment errors or from spliced noncoding genes. Overall, candidate introns had an average multiplicity of 7898. 1403 candidate introns had a multiplicity of one while 3362 had a multiplicity smaller or equal to five. OGS3 contains about 30\% more RNA-Seq supported introns than OGS2: 41,921 out of 54,909 introns in OGS2 (76.3\%) and 54,513 out of 63,211 in OGS3 (86.2\%) are identical to an intron suggested by RNA-Seq spliced read alignments (Table 4 ).

\section{BUSCO analysis reveals very high accuracy of the gene set}

The completeness of OGS3 was assessed using BUSCO (Benchmarking Universal Single-Copy Orthologs) and compared to the value for OGS2 [44] and to those of other sequenced genomes [45-47]. The genome of Drosophila melanogaster can be assumed to be the best annotated genome of insects, the genome of Apis mellifera was recently re-annotated and is therefore comparable to the OGS3 from Tribolium and for Parasteatoda tepidariorum, for which the first genome version was just published with the peculiarity of large duplication events. Nearly all of the conserved genes from the BUSCO Arthropoda set where found in OGS2 and OGS3 (Table 5). OGS3 (99.6\%) scored slightly better than OGS2 (99.3\%). The completeness of OGS3 rivals 
Table 4 Annotation improvement

\begin{tabular}{lll}
\hline & OGS2 & OGS3 \\
\hline Number of genes & 16,561 & 16,593 \\
Average coding length & $1341 \mathrm{bp}$ & $1473 \mathrm{bp}$ \\
Number of coding exons per transcript & 4.32 & 5.02 \\
GC content & $0.4597 \%$ & $0.4625 \%$ \\
Fraction of single exon genes & $17.66 \%$ & $17.74 \%$ \\
Number of introns (excluding UTR) & $54,909(54875)$ & $63,211(58837)$ \\
Fraction of RNA-Seq-supported introns & $76.3 \%$ & $86.2 \%$ \\
Average intron length & $1167 \mathrm{bp}$ & $1362 \mathrm{bp}$ \\
\hline
\end{tabular}

that of Drosophila (99.8\%) and is better than Apis (97.9\%) or Parasteatoda (94.4\%) (Table 5).

\section{Official gene set and NCBI RefSeq genome}

The genome assembly as well as the gene models have been submitted to Genbank (NCBI) as the RefSeq genome (GCF_000002335.3) and Tribolium (OGS3) (GCA 000002335.3) [48]. Genome assembly 5.2 and gene set OGS3 are available on the NCBI website (ftp://ftp.ncbi. nlm.nih.gov/genomes/all/GCF/000/002/335/GCF 000002335.3_Tcas5.2) and are available as a preselection in several NCBI services, such as the BLAST search.

\section{Protein sequence conservation}

Drosophila melanogaster and Caenorhabditis elegans are the main invertebrate models for functional genetics and have contributed tremendously to the understanding of cellular and molecular processes relevant for vertebrate biology. However, their protein sequences are quite diverged compared to Apis mellifera or the annelid Platynereis dumerilii [49]. The transferability of findings to other taxa may depend, among other things, on the biochemical conservation of the proteins involved. Hence, when choosing a model system, the conservation of the proteome is an important aspect. In Tribolium, the genetic toolkit is more developed compared to other insects (except for Drosophila) or annelids. Unbiased genomewide screening has been established making Tribolium an excellent alternative model for studying basic biological processes. We therefore asked how the protein sequences of the red flour beetle compare to other invertebrate model systems. As outgroup we used the main vertebrate model organism for medical research, the mouse Mus musculus.

We identified 1263 single-copy orthologs across five species, made an alignment and calculated a phylogenetic tree (Fig. 1a). The Tribolium branch is shorter compared to those of Drosophila and C. elegans indicating that the Tribolium proteome is more similar to that of the mouse than are the proteomes of Drosophila and Caenorhabditis. In this comparison the annelid proteome appears to be even more similar to that of the mouse proteome. In such alignment-based sequence comparisons, the less conserved non-aligneable parts of the proteins are not considered. Therefore, we used an alignment-free method for measuring sequence distances $[50,51]$ on the same dataset and found it to basically reflect the same conclusion albeit with less resolution (Fig. 1b).

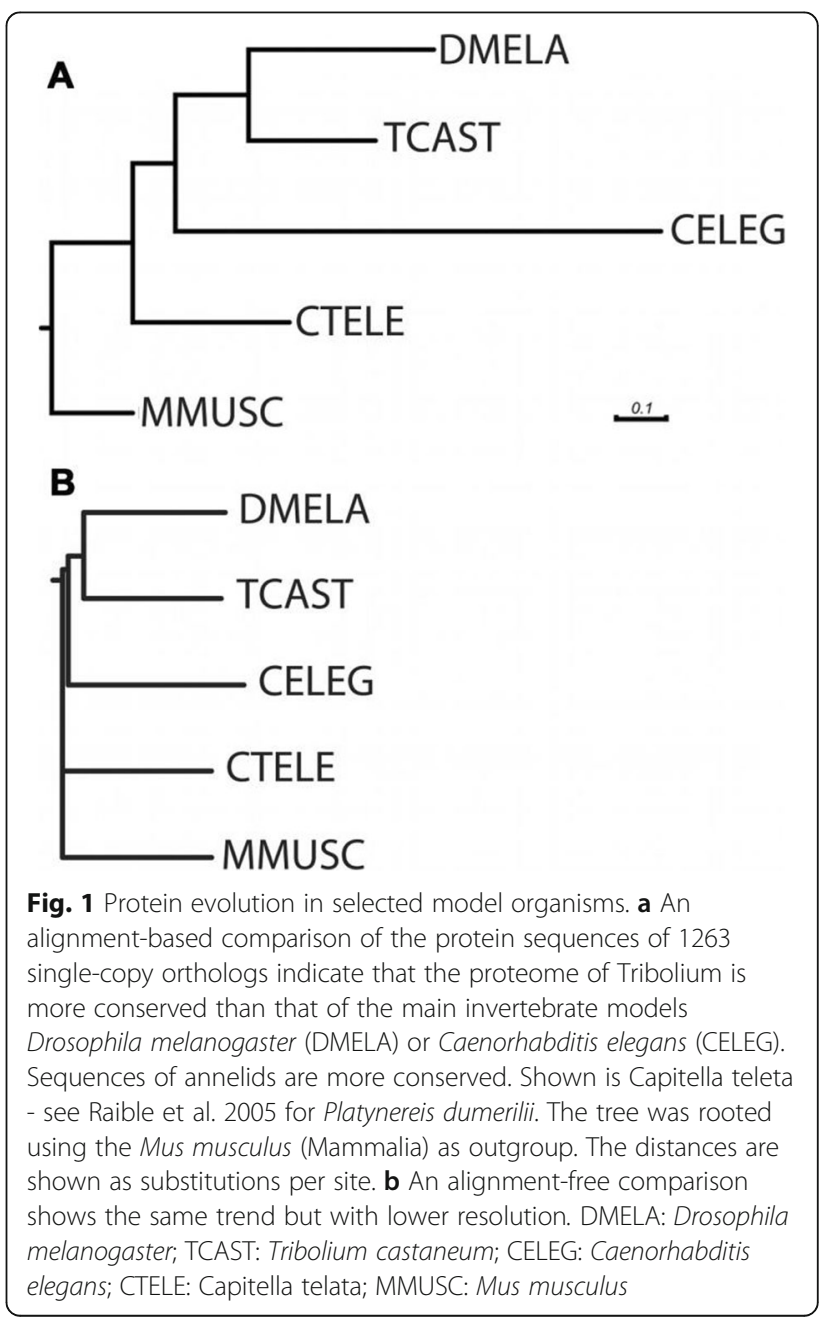




\section{Prediction of microRNA binding sites}

MicroRNAs are short non-coding RNAs that regulate gene expression by guiding the RNA-induced silencing complex (RISC) to complementary sites in the 3'UTR regions of target mRNAs (reviewed in [52]). The principal interaction between microRNAs and their targets occurs through the so-called "seed" region, corresponding to the 2nd and 8th position of the mature microRNA sequence [53], and this complementarity can be used for computational predictions of microRNA-target pairs. Previous studies experimentally identified 347 microRNA genes in the Tribolium castaneum genome, each of which can generate two mature microRNAs derived from the two arms (5p and 3p) of the microRNA precursor hairpin (Additional file 1: Table S1) [54, 55]. We extracted the 3'UTR sequences of Tribolium proteincoding genes and annotated potential microRNA binding sites in these regions using an algorithm based on the microRNA target recognition principles described in [53]. In addition, we generated an alternative set of computational microRNA target predictions using an algorithm based on the thermodynamic properties of microRNA-mRNA duplexes irrespective of seed complementarity [56]. The two algorithms identified 309,675 and 340,393 unique putative microRNA-target pairs, with approximately $60 \%$ overlap. Moreover, a similar number of genes in each set, 13,136 and 13,057 respectively, had at least one microRNA target site.

\section{Comparison of microRNA target gene sets}

MicroRNAs are recognized as important players in animal development, and their role in insects is best understood in the classical model organism Drosophila melanogaster. Comparative genomic analyses showed that 83 Tribolium castaneum microRNAs have one or more homologs in Drosophila [54, 55]. To assess whether conserved microRNAs also have a conserved target repertoire, we sought to assess the number of orthologous genes targeted by each conserved microRNA pair. To this end, we used an identical target prediction approach to determine microRNA-target pairs in Drosophila melanogaster, and calculated the numbers of homologous and non-homologous targets for each conserved microRNA pair in the two species (Additional file 1: Table S1). Results indicated that even though the majority of homologous microRNAs have conserved seed sequences for at least one mature product, their target repertoires diverged.

Nonetheless, a subset of well-conserved microRNAs had higher numbers of common predicted targets than expected by chance, especially based on seed complementarity. These included members of the bantam, mir184, 279/miR-996, mir-2/11/13/2944/6, mir-9, mir-14, mir-1, mir-7, mir-34 seed families, which have been previously identified for their roles in key developmental processes in Drosophila, and are highly expressed in both fruit fly and beetle embryos.

Given the large number of target predictions identified for individual microRNAs we examined the specific conserved targets for one of the microRNAs that both exhibited significant target conservation and had well characterized targets in Drosophila. The miR-279/miR996 family has been extensively characterized for its role in regulating the emergence of $\mathrm{CO} 2$ sensing neurons and in circadian rhythms. in Tribolium, of the nine characterized targets identified in Drosophila, one had no clear ortholog $(u p d)$, four did not have conserved targeted sequences in their UTRs (STAT, Rho1, boss, and gcm), but four targets (nerfin-1, esg, ru, and neur) had strongly conserved predicted target sites. microRNA regulation of all these four targets has clear functional importance in these developmental processes and two of them (nerfin-1 and esg) work together as key players in the formation of $\mathrm{CO}_{2}$ sensing neurons [57].

In summary, we provide an example where conserved microRNA regulate similar developmental pathways between the two taxa. It will be interesting to determine the degree of conservation of the entire microRNA set. The predicted microRNA binding sites are now available as tracks in the genome browser at iBeetle-Base (https:// ibeetle-base.uni-goettingen.de/gb2/gbrowse/tribolium/).

\section{Discussion}

With respect to the toolkit for functional genetics in insects, the red flour beetle Tribolium castaneum is second only to Drosophila melanogaster. The work described here focused on enhancing genomic resources to support functional genetic work in Tribolium castaneum. To that end we increased the contiguity of the genome assembly and generated a significantly improved OGS by adding novel information such as splice variants and microRNA target sites.

In order to close gaps and place more contigs on scaffolds, we added data from long-insert jumping libraries and BioNano Genomics optical mapping. It turned out that the latter contributed much more to enhance the previous assembly based on Sanger sequencing: While the first approach increased the N50 by $20 \%$ the BioNano Genomics consensus mapping led to another 3fold increase of the N50. Hence, data from large single molecules is best suited to overcome the limits of sequencing-based assemblies. Compared to the recently re-sequenced genome assembly of the honey bee [46] our scaffold N50 is significant higher (4753 kb compared to $997 \mathrm{~kb})$. This is also true for the number of placed contigs (2149 compared to 5645). However, compared to Drosophila, the most thoroughly sequenced insect 
genome (contig N50 19,478 kb), our improved assembly still lags behind.

The improved genome assembly and extensive RNASeq data provided the basis for an enhanced gene prediction. The BUSCO values indicate a more complete OGS, closer to Drosophila than to other emerging model insects. Further, 11\% more RNA-Seq reads could be mapped to the gene predictions of OGS3 compared to OGS2, which is a relevant increase e.g. for differential gene expression analyses. The overall number of genes did not increase much. On one hand, 1452 genes without sequence similarity to OGS2 were newly added to the gene set. On the other hand, a similar number of genes from OGS2 is not represented in OGS3. These were mostly very short genes not supported by RNA-Seq data. Hence, most of them were probably false predictions in the former gene set.

Qualitative enhancement includes the detection and annotation of alternative splice variants. Since RNAi is splice variant specific in Tribolium [58], this opens the possibility to systematically check for differences in the function of isoforms. Further, the inclusion of UTR regions for many more genes enabled us for the first time to comprehensively map candidate microRNA binding sites to our gene set. Indeed, we have identified a large number of microRNA target sites in orthologs of both Drosophila and Tribolium. The microRNAs that we identified to have conserved targets belong mostly to microRNA families where obvious loss-of-function phenotypes have previously been characterized in other animals. One example is the miR-279/miR-996 family that share a common seed and have been found to play a key role in Drosophila $\mathrm{CO} 2$ sensing neurons and ovarian border cell development [57]. A number of the key microRNA targets identified in Drosophila, such as nerfin, escargot, and neuralized were predicted to be targets of Tribolium miR-279. This striking example of conservation illustrates that further comparative approaches have the potential to identify conserved regulatory networks involving microRNAs within insects based on the resources provided here. Enhanced coverage with RNA data revealed the transcription start sites of most genes, which helps in the design of genome editing approaches and of transgenic constructs based on endogenous enhancers and promoters $[22,23,35,59]$.

Finally, we show that the proteome of Tribolium is less diverged from the vertebrate proteome than that of Drosophila, which is an argument for using Tribolium as alternative model system when the biochemical function of proteins with relevance to human biology is studied.

\section{Conclusions}

The new genome assembly for Tribolium castaneum and the respective gene prediction is available at NCBI as a RefSeq genome and a new official gene set (OGS3). This promotes functional genetics studies with respect to a plethora of topics in Tribolium, opens the way for further comparative genomics, e.g. with respect to microRNAs, and positions Tribolium as a central model organism within insects.

\section{Methods \\ Genome resequencing and assembly Reference genome files}

The $T$. castaneum reference genome assembly (Tcas_3.0, NCBI accession number AAJJ01000000) was downloaded from NCBI. The following 23 contigs, which had been marked by NCBI as contaminants were removed: AAJJ01000455, AAJJ01001129, AAJJ01001336, AAJJ01001886, AAJJ01003084, AAJJ01003125, AAJJ01003874, AAJJ01004029, AAJJ01004493, AAJJ01004617, AAJJ01005150, AAJJ01005727, AAJJ01005755, AAJJ01006305, AAJJ01006331, AAJJ01007110, AAJJ01007612, AAJJ01007893, AAJJ01008452, AAJJ01009546, AAJJ01009593, AAJJ01009648, and AAJJ01009654. In addition, the first 411 nucleotides from AAJJ01009651, and the first 1846 and last 46 nucleotides from AAJJ01005383 were removed after being identified as contaminants. The remaining 8815 contigs $(\mathrm{N} 50=43 \mathrm{~Kb})$ had been used to construct the 481 scaffolds $(\mathrm{N} 50=975 \mathrm{~Kb})$ included in Tcas 3.0. Information from a genetic recombination map based on molecular markers [39], was used to anchor 176 scaffolds in 10 superscaffolds (often referred to as pseudomolecules or chromosome builds). In Tcas 3.0 these are referred to as ChLGX and ChLG2-10, representing the linkage groups in the recombination map. The remaining 305 scaffolds and 1839 contigs that did not contribute to the superscaffolds were grouped together in Beetlebase (http://beetlebase.org or $\mathrm{ftp}: / / \mathrm{ftp}$. bioinformatics.ksu.edu/pub/BeetleBase/3.0/Tcas_3.0_ BeetleBase3.0.agp) (unknown placement).

Table 5 BUSCO analysis

\begin{tabular}{|c|c|c|c|c|c|}
\hline & $\begin{array}{l}\text { Tcas } \\
\text { OGS2 }\end{array}$ & $\begin{array}{l}\text { Tcas } \\
\text { OGS3 }\end{array}$ & $\begin{array}{l}\text { Dmel } \\
\text { r16.19 }\end{array}$ & Amel 4.5 & Ptep 2.0 \\
\hline Complete & $\begin{array}{l}1058 \\
(99.3 \%)\end{array}$ & $\begin{array}{l}1061 \\
(99.6 \%)\end{array}$ & $\begin{array}{l}1063 \\
(99.8 \%)\end{array}$ & $\begin{array}{l}1043 \\
(97.9 \%)\end{array}$ & $\begin{array}{l}1007 \\
(94.4 \%)\end{array}$ \\
\hline $\begin{array}{l}\text { Complete single } \\
\text { copy }\end{array}$ & $\begin{array}{l}1054 \\
(98.9 \%)\end{array}$ & $\begin{array}{l}1056 \\
(99.1 \%)\end{array}$ & $\begin{array}{l}1055 \\
(99 \%)\end{array}$ & $\begin{array}{l}1038 \\
(97.4 \%)\end{array}$ & $\begin{array}{l}966 \\
(90.6 \%)\end{array}$ \\
\hline $\begin{array}{l}\text { Complete } \\
\text { duplicated }\end{array}$ & $4(0.4 \%)$ & $5(0.5 \%)$ & $8(0.8 \%)$ & $5(0.5 \%)$ & $41(3.8 \%)$ \\
\hline Fragmented & $5(0.5 \%)$ & $2(0.2 \%)$ & $0(0 \%)$ & 15 (1.4\%) & $18(1.7 \%)$ \\
\hline Missing & $3(0.2 \%)$ & $3(0.2 \%)$ & $3(0.2 \%)$ & $8(0.7 \%)$ & $41(3.9 \%)$ \\
\hline $\begin{array}{l}\text { Genes in BUSCO } \\
\text { profile }\end{array}$ & 1066 & 1066 & 1066 & 1066 & 1066 \\
\hline
\end{tabular}


Table 6 Mate pairs jumping library statistics

\begin{tabular}{llr}
\hline FastQ & Total reads & Total length \\
\hline 3kb_1 & $23,677,983$ & $2,120,896,823$ \\
3kb_2 & $23,677,983$ & $2,123,186,604$ \\
8kb_1 & $23,202,365$ & $2,093,651,921$ \\
8 kb_2 & $23,202,365$ & $2,096,015,114$ \\
20kb_1 & $12,884,671$ & $1,151,209,160$ \\
20kb_2 & $12,884,671$ & $1,153,515,873$ \\
\hline
\end{tabular}

\section{Description of Illumina libraries}

The DNA used to construct three long-insert jumping libraries (3, 8, and $20 \mathrm{~kb}$ target size) was isolated at the Baylor Human Genome Sequencing Center in 2004 for Sanger-based sequencing. Thus, the source of DNA for these data is the same as for the original reference genome. The insert sizes for the three libraries are $3173 \mathrm{bp}$, $6775 \mathrm{bp}$, and $34,825 \mathrm{bp}$, respectively, with $10-15 \%$ standard deviation. Library construction, Illumina sequencing and cleaning were performed by MWGOperon (Europe). For all libraries, reads of minimum length $30 \mathrm{bp}$ and maximum $100 \mathrm{bp}$ were retained after cleaning and removal of the internal spacer. The "_1" files contain the forward reads while the "_2" files contain the reverse reads. Reads lacking the spacer or containing insert sequence only on one side of the spacer were not used. Table 6 lists the number of reads and their length for the jumping libraries.

\section{Scaffolds linked with atlas-link v0.01}

Atlas-Link is a software tool that links and orients scaffolds using mate pair libraries (www.hgsc.bcm.edu/software/atlas-link). Scaffolds in the original assembly (Tcas3.0) were indexed using the IS algorithm in BWA prior to running Atlas-Link on each long insert jumping library with the settings described in Additional file 2 . Table 7 shows the improvements that were achieved by Atlas-Link. Scaffold order and placement within Chromosome LG builds was used to validate the Atlas -Link output. We used a value of 300 minimum links, which reproduced most of the original order, linking neighboring scaffolds and adding scaffolds that were unplaced in Tcas 3.0. The output AGP file, was renumbered to reflect the NCBI coordinates. Detailed steps and scripts are provided in Additional file 2.

\section{Contigs extended and gaps closed with GapFiller v1.10}

We used the sequence data from the jumping libraries to fill small gaps in the original assembly. Running GapFiller v1.10 to 20 iterations with strict parameters (detailed parameters, and scripts are provided in Additional file 2).

\section{Scaffolds joined using BioNano genomics consensus maps}

The genome assembly output from GapFiller was used to generate in silico maps for comparison to BioNano consensus maps and refered to as Tcas5.0 in [41]. Table 8 displays the number, length and N50 of the scaffolds before and after consensus mapping.

Table 7 Number of scaffolds and ungapped length before and after running Atlas-Link

\begin{tabular}{lllllll}
\hline Molecule & $\begin{array}{l}\text { Scaffolds } \\
\text { before }\end{array}$ & $\begin{array}{l}\text { Ungapped length } \\
\text { before }\end{array}$ & $\begin{array}{l}\text { Scaffolds } \\
\text { after }\end{array}$ & $\begin{array}{l}\text { Ungapped length } \\
\text { after }\end{array}$ & $\begin{array}{l}\text { Unplaced scaffolds } \\
\text { added }\end{array}$ & $\begin{array}{l}\text { Unplaced ungapped } \\
\text { length added }\end{array}$ \\
\hline LG1 X & 13 & $7,011,684$ & 13 & $7,071,107$ & 2 & 59,423 \\
LG2 & 20 & $14,013,343$ & 18 & $14,229,660$ & 2 & 216,317 \\
LG3 & 35 & $27,022,651$ & 29 & $28,072,007$ & 8 & $1,049,356$ \\
LG4 & 7 & $11,540,046$ & 6 & $11,540,046$ & - & - \\
LG5 & 17 & $13,832,902$ & 17 & $14,111,830$ & 3 & 278,928 \\
LG6 & 15 & $8,229,537$ & 12 & $8,262,430$ & 2 & 32,893 \\
LG7 & 18 & $14,841,431$ & 15 & $15,084,119$ & 3 & 242,688 \\
LG8 & 16 & $12,760,817$ & 14 & $12,870,760$ & 1 & 109,943 \\
LG9 & 21 & $14,567,469$ & 21 & $14,900,846$ & 2 & 333,377 \\
LG10 & 14 & $7,043,942$ & 12 & $7,070,154$ & 1 & 26,212 \\
Unplaced multi-contig & 305 & $16,272,476$ & 263 & $14,079,574$ & & \\
Unplaced single-contig & 1839 & $4,176,957$ & 1820 & $4,020,722$ & & \\
Total & 2320 & $151,313,255$ & 2240 & $151,313,255$ & & \\
\hline
\end{tabular}


Table 8 Number of scaffolds, scaffolds' length, and N50 before and after using BNG consensus maps

\begin{tabular}{llllllll}
\hline Molecule & Scaffolds before & Scaffolds after & Length (Mb) before & Length (Mb) after & N50 (kb) before & N50 (kb) after & Unplaced scaffolds added \\
\hline LG1 $=$ X & 13 & 4 & 7.34 & 8.92 & 1160.70 & 7264.05 & 2 \\
LG2 & 18 & 8 & 14.78 & 15.034064 & 1207.76 & 9314.472 & 0 \\
LG3 & 29 & 18 & 29.78 & 31.017975 & 1409.81 & 2672.697 & 3 \\
LG4 & 6 & 3 & 12.11 & 12.24 & 2906.70 & 9484.15 & 0 \\
LG5 & 17 & 7 & 14.64 & 15.36 & 1402.64 & 4484.65 & 1 \\
LG6 & 12 & 9 & 9.02 & 9.25 & 956.12 & 2189.88 & 0 \\
LG7 & 15 & 6 & 15.74 & 16.48 & 1333.70 & 8809.74 & 0 \\
LG8 & 14 & 9 & 13.66 & 13.98 & 1312.85 & 4002.45 & 1 \\
LG9 & 21 & 10 & 15.81 & 16.12 & 893.90 & 4920.63 & 0 \\
LG10 & 12 & 11 & 7.54 & 8.84 & 1198.49 & 1224.30 & 3 \\
Unplaced & 2083 & 2072 & 20.33 & 17.35 & 150.43 & 104.32 & 2 \\
Total & 2240 & 2157 & 160.74 & 164.60 & 1160.70 & 4002.45 & 12 \\
\hline
\end{tabular}

\section{Annotation}

The reannotation of the protein-coding genes of Tribolium castaneum was done in three main steps: 1) automatic gene prediction based on an unpublished intermediate assembly 4.0 with AUGUSTUS [42] incorporating evidence from multiple sources, 2) merging the gene prediction with the previous official gene set OGS2 [37] and 3) a mapping of the new gene set to assembly 5.2 using liftover [60]. Additionally, manual curation and correction was completed for 399 genes. The RNA-seq reads collected in this project are submitted under Bioproject PRJNA275195 (https://www.ncbi.nlm.nih.gov/ bioproject/PRJNA275195).

\section{Protein-coding genes}

AUGUSTUS is a gene prediction tool based on a hidden Markov model that allows one to incorporate extrinsic evidence such as from RNA-Seq or protein homology. Such extrinsic evidence is summarized in the form of so-called 'hints' that are input to AUGUSTUS and that represent mostly soft evidence on the location of exons, introns and other gene features.

RNA-Seq libraries of around 6.66 billion reads from the iBeetle consortium and 9 external contributors constitute the majority of evidence. All reads were aligned against the repeat masked genome assembly 4.0 with GSNAP [61]. Hits were filtered according to three criteria. A hit must reach a minimum identity threshold of 92\%. Furthermore, a paired read filter was applied: Reads that are paired must not exceed a genomic distance of $200 \mathrm{Kbp}$ and must be correctly oriented towards each other. Subsequently, reads that could not be unambiguously aligned to a single locus (the identities of the two highest-scoring alignments were within $4 \%$ of each other) were discarded in order to avoid false positives such as from pseudogenes.
It is often hard to correctly align spliced reads, especially when they are spliced near the beginning or end of the read. Therefore, an iterative mapping approach was applied. First a set of preliminary introns was generated by using the spliced alignments found by GSNAP and by predicting introns ab initio with AUGUSTUS. Removing sequences of these introns produced partial spliced transcripts to which all reads were aligned a second time. We obtained an improved spliced alignment set with additional spliced alignments via a coordinate change induced by the coordinates of the preliminary introns (http://bioinf.uni-greifswald.de/bioinf/wiki/pmwiki.

php? $n=$ IncorporatingRNAseq.GSNAP). From the gaps in the read alignments hints on the location of introns were compiled, including the number of reads that support each intron. Further, from the RNA-Seq genome coverage hints on the location of (parts of) exons were generated.

In addition, evidence from 64,571 expressed sequence tags (ESTs), 19,284 proteins of invertebrates (from uniprot/swissprot database), repetitive regions in the genome detected by RepeatMasker (Smit, AFA, Hubley, R \& Green, P. RepeatMasker Open-4.0.2013-2015, http:// www.repeatmasker.org), 387 published coding genes from NCBI, 69 odorant binding Proteins [62] and 60 "gold standard" sequences that derived from single gene sequence analyses by different groups of the Tribolium community. The RNA-Seq reads are available at public databases in the Bioproject PRJNA275195.

\section{Integration of the previous gene set}

Several analyses indicated that the AUGUSTUS gene set is more accurate. First, a higher number of RNA-seq reads mapped to the OGS3 compared to OGS2. Second, a large portion of genes that are present in OGS3 but not OGS2 were confirmed by additional evidence like blast hit or RNA-seq coverage. Third, most of the genes 
present in OGS2 but "lost" from OGS3 lacked such additional evidence indicating that they had been false positive annotations of OGS2. However, unclear loci remain, in which the true annotation is yet unknown. In order to introduce some stability in the gene set update we kept the old genes when in doubt whether a newly predicted gene with another structure is indeed a correction of the old gene structure. We address the problem of finding such gene structures by introducing the concept of specifically supported genes. Consider a gene $g_{\mathrm{OGS} 2}$ from the previous gene set and a set of overlapping genes $G_{\mathrm{AUG}}$ from the AUGUSTUS prediction. $g_{\mathrm{OGS} 2}$ is said to be specifically supported, if it has at least one intron supported by RNA-Seq, that none of the genes in $G_{\text {AUG }}$ have. Additionally, every supported intron of genes in $G_{\mathrm{AUG}}$ is also in $g_{\mathrm{OGS2}}$. In OGS3 we kept all specifically supported OGS2 genes and discarded all AUGUSTUS genes overlapping them.

The set of supported intron candidates was compiled from spliced RNA-Seq reads with a number of restrictions. Each intron candidate had to have a length between 32 and $350,000 \mathrm{bp}$, all splice sites had to be contain the appropriate sequences and the number of hints supporting a contradicting gene structure had to be at most 9 times higher than the number of hints supporting the intron candidate itself.

Additionally, we kept an OGS2 gene that did not overlap any AUGUSTUS gene, if it had homologs in Drosophila or other invertebrates or an annotated function (GO term listed in the Gene Ontology database [63]) or was covered by RNA-Seq reads with FPKM $\geq 0.01$ (calculated with eXpress [64]). In total we kept 3087 OGS2 genes and 13,413 AUGUSTUS genes.

\section{Liftover from assembly 4.0 to assembly 5.2}

After a Tribolium community call many genes were manually reviewed and edited based on an intermediate assembly 4.0. To preserve manually curated gene structures, we decided to transfer the new gene set to assembly 5.2. We created an assembly map that assigns each base of assembly 4.0 to a base in the new assembly 5.2 , if possible. This map file was used to 'lift' above gene set to the updated assembly 5.2 using liftOver taken from the UCSC Genome Toolbox (http://hgdownload.cse.ucsc.edu/admin/exe/linux. x86_64.v287/). 337 genes could not be unambiguously and completely mapped. We applied our annotation pipeline to the new assembly and merged the result with the lifted gene set from the previous assembly. Consequently, we were able to identify gene structures for which the improved assembly allowed a better annotation. The new gene set was complemented by 469 gene structures that could only be predicted based on the new assembly. Furthermore, we corrected 745 of the lifted gene structures according to the concept of specific supported genes as described above.

The standard Viterbi algorithm used in AUGUSTUS predicted 159 transcripts with an in-frame stop codon spliced by an intron. To replace them with alternative gene structures that do not contain in-frame stop codons we ran AUGUSTUS with the option - mea $=1$ on the affected regions. MEA is an alternative algorithm that can prohibit spliced in-frame stop codons but needs more computational time. During the GenBank submission process some gene models were revised and seven genes were manually edited or deleted based on suggestions from NCBI.

\section{Orthology assignment and proteome analyses}

Orthologs and paralogs between $T$. castaneum and $D$. melanogaster were found using the OrthoDB database [65] and results were formatted accordingly using custom Perl scripts.

For the phylogenetic analysis, we compared T. castaneum (Insecta:Coleoptera) with three other invertebrates; Drosophila melanogaster (Insecta:Diptera), Caenorhabditis elegans (Nematoda) and Capitella teleta (Annelida). The mammalian Mus musculus was used as outgroup. More specifically, we used OrthoDB and obtained 1263 single-copy orthologs, in order to perform a phylogenomics analysis with RAxML [66]. Briefly, a multiple sequence alignment was built for each orthologous group separately, using MUSCLE [67]. Then, the resulting alignments were trimmed using trimAl [68] with parameters "-w 3 -gt 0.95 -st 0.01 " and concatenated using custom Perl scripts. The concatenated alignment was subsequently used to perform a phylogenomic analysis using RAxML 7.6.6 (PROTGAMMAJTT model of amino acid substitutions) with 100 bootstrap replicates. The final tree was edited with EvolView [69] and InkScape 0.91.

The same set of genes was analyzed separately in an alignment independent approach (see Additional file 2 for details). Two approaches were performed using six distance measures $(\mathrm{d} 1, \ldots, \mathrm{d} 6)$ : In the first approach, we used 'gdist' to determine the pairwise distances between sequences inside the groups, then 'phylip neighbor' to compute corresponding phylogenetic trees, rooted by setting MMUSC as outgroup, and computing the consensus tree using 'phylip consense'. In the second approach, we concatenated sequences in the groups in random order to form five artificial "whole proteom" sequences (one for each of the species), determined their pairwise distances and computed a phylogenetic tree using 'phylip neighbor', again setting the MMUSC sequence as outgroup. To check for robustness of the approach and also the influence of sequence lengths 
we performed these experiments with different subsets: (1) with all 1263 groups and (2) with a subset of the all groups. The subsets we considered were: (2a) groups with a certain minimum sequence length, $(2 b)$ only groups whose sequence lengths differed by at most a certain percentage, and (2c - only for experiment (B)) a random selection of groups (for instance, randomly select $80 \%$ of all groups for concatenation). Concatenation experiment (B) produced phylogenies that turned out to be almost immune against changes in order of concatenation and considerably robust against restricting consideration to all groups or subsets of groups concatenation. Best signals where obtained by distance $\mathrm{d} 6$, which resulted in the phylogeny displayed in Fig. $1 \mathrm{~b}$.

\section{microRNA prediction}

Mature sequences of $T$. castaneum microRNAs (Additional file 1) were retrieved from previous annotations $[54,55]$, and D. melanogaster microRNAs were retrieved from miRBase v21 [70]. D. melanogaster transcript 3'UTR sequences were retrieved from Flybase r6.09 [71]. MicroRNA target predictions in the two species were performed using two independent approaches. First, we identified target transcripts having regions complementary to the microRNA 7A1, $7 \mathrm{~m} 8$ and 8 mer seed sequences as described in [53] using a custom script provided by Antonio Marco [54], and the miRanda and TargetScan algorithms [56, 72], with default parameters. Previously established conserved microRNAs between $T$. castaneum and D. melanogaster $[54,55]$ were used to assess conserved microRNA-target pairs. For microRNAs with more than 1 homolog in the other species, we assessed all possible combinations of homologous pairs. The numbers of conserved microRNA-target interactions (homologous microRNAs targeting homologous genes) were calculated using a custom script. The significance of the conserved target pair numbers was assessed by comparison with the number of orthologous genes obtained by random sampling of equal size without replacement 1000 times.

\section{Supplementary information}

Supplementary information accompanies this paper at https://doi.org/10. 1186/s12864-019-6394-6.

Additional file 1: Table S1. Table summarizing microRNA data

Additional file 2. Details and scripts used for genome assembly and alignment free phylogenetic tree construction.

\section{Abbreviations}

BLAST: Basic local alignment search tool; BLAT: BLAST like alignment tool; bp: base pairs; BUSCO: Benchmarking Universal Single-Copy Orthologs; CDS: Coding sequence; EST: Expressed sequence tag; LG: Linkage group; Mb: Megabases; mRNA: Messenger RNA; OGS3: Official gene set version 3; RNAi: RNA interference; RNA-Seq: Next generation sequencing of mRNAs;
Tcas5.2: Official assembly of genomic sequence of Tribolium castaneum version 5.2; UTR: Untranslated region

\section{Acknowledgements}

We thank Jiaxin Qu, Xing-Zhi Song, Xiang Qin, Kim Worley, and Stephen Richards at HGCS, BCM supplying the DNA and for instruction on running Atlas link.

\section{Authors' contributions}

$\mathrm{NH}$, JS and SJB performed the genome assembly, analyzed the data and drafted the manuscript. LG and MSt performed the annotation, analyzed the data, determined the new official gene set and drafted the manuscript. PI, RMW, EZ performed the orthology assignment of the new gene set and drafted the manuscript. MN, SGJ and MR annotated the microRNA binding sites and drafted the manuscript. JD analyzed data and drafted the manuscript. $C L$ determined the termini of the gene annotations. CD performed the alignment free sequence comparison and drafted the manuscript. JS, PK, JU, SD, GO, YH, JS, MS, SL, AM, NP, DG, TH, JS, IMVJ, KAP performed the manual quality control of the new gene set. JL, EAW, DS, SR, RS, YP,MS, MK, HRC, SK MF, BA, AV contributed sequencing data. MSt, SJB and GB conceived of and coordinated the project and drafted the manuscript. All authors consent with the publication of this version of the manuscript. All authors read and approved the final manuscript.

\section{Funding}

We thank the Max-Planck-Society for financial support via the department of Dr. Herbert Jäckle and for the help by Dr. Ulrike Löhr.

$\mathrm{SJB}, \mathrm{NH}$ and JS were supported by an Institutional Development Award (IDeA) from the National Institute of General Medical Sciences of the National Institutes of Health under grant number P20 GM103418. The content is solely the responsibility of the authors and does not necessarily represent the official views of the National Institute of General Medical Sciences or the National Institutes of Health.

GB, JD, MS, MK and EAW were supported by the iBeetle project (DFG research unit FOR1234; BU1443/6, BU1443/7, BU1443/8, STA 1009/10, KL 656/ 7, Scho1058/4, WI1797/5).

DS was supported by a BIF PhD fellowship from Boehinger Ingelheim Fonds; SR was funded by the SFB680 (DFG).

DG, TH, JS, IMVJ, and KAP were supported by DFG grants PA 2044/1-1 and SFB 680 Project A12 to KAP.

RMW was supported by Swiss National Science Foundation grant PP00P3_170664

The funders had no role in design of the study, interpretation and publication of the data.

\section{Availability of data and materials}

The datasets generated and analyzed during the current study are available in the following repositories:

The RefSeq genome assembly 5.2 (GCF 000002335.3) and the official gene set for Tribolium castaneum (OGS3) (GCA_000002335.3) are available at Genbank (NCBI). (Genbank: https://www.ncbi.nlm.nih.gov/genome/?term= GCA_000002335.3; ftp download: ftp://ftp.ncbi.nlm.nih.gov/genomes/all/GCF/ 000/002/335/GCF_000002335.3_Tcas5.2) and at iBeetle-Base: https://ibeetlebase.uni-goettingen.de/help/resources

The RNA-Seq reads are available at public databases in the Bioproject PRJNA275195 (https://www.ncbi.nlm.nih.gov/bioproject/PRJNA275195). The data and software underlying the alignment free sequence comparison is found in the following repository https://hdl.handle.net/21.11101/00000007-D64E-1. It contains: sequence data of the single-copy orthologs; executables of the used software (along with the source code; a jupyter notebook to execute the analysis we have done and a README file.

Ethics approval and consent to participate Not relevant.

\section{Consent for publication \\ Not relevant.}

Competing interests

Robert M. Waterhouse is Editorial Board Member at BMC Genomics. Otherwise, there are no competing interests 


\section{Author details}

'Department of Computer Science, East Carolina University, Greenville, NC 27858, USA. ${ }^{2}$ Division of Biology, Kansas State University, Manhattan, KS 66506, USA. ${ }^{3}$ Institut für Mathematik und Informatik, Universität Greifswald, Greifswald, Germany. ${ }^{4}$ Department of Genetic Medicine and Development, University of Geneva Medical School and Swiss Institute of Bioinformatics, 1211 Geneva, Switzerland. ${ }^{5}$ Faculty of Biology, Medicine and Health, University of Manchester, Michael Smith Building, Oxford Road, Manchester M13 9PT, UK. ${ }^{6}$ Department of Evolutionary Developmental Genetics, GZMB, University of Göttingen, Justus-von-Liebig-Weg 11, 37077 Göttingen, Germany. ${ }^{7}$ Department of Ecology and Evolution, University of Lausanne and Swiss Institute of Bioinformatics, 1015 Lausanne, Switzerland. ${ }^{8}$ Department of Biology, Miami University, Oxford, OH 45056, USA. ${ }^{9}$ Institut für Informatik, Fakultät für Mathematik und Informatik, Georg-August-Universität Göttingen, Goldschmidtstr. 7, 37077 Göttingen, Germany. ${ }^{10}$ Göttinger Graduiertenschule fur Neurowissenschaften Biophysik und Molekulare Biowissenschaften, Georg-August-Universität Göttingen, Göttingen, Germany. ${ }^{11}$ Department of Developmental Biology, GZMB, University of Göttingen, Justus-von-Liebig-Weg 11, 37077 Göttingen, Germany. ${ }^{12}$ Institute for Zoology: Developmental Biology, University of Cologne, Zülpicher Str. 47b, 50674 Cologne, Germany. ${ }^{13}$ School of Life Sciences, University of Warwick, Gibbet Hill Campus, Coventry CV4 7AL, UK. ${ }^{14}$ Department Developmental Biology, GZMB, University of Göttingen, Justus-von-Liebig-Weg 11, 37077 Göttingen, Germany. ${ }^{15}$ Department of Developmental Biology, University of Göttingen, Justus-von-Liebig-Weg 11, 37077 Göttingen, Germany. ${ }^{16}$ Institute of Zoology: Developmental Biology, University of Cologne, Zülpicher Weg 47b, 50674 Cologne, Germany. ${ }^{17}$ Institut für Biowissenschaften, Universität Rostock, Albert-Einstein-Str. 3, 18059 Rostock, Germany. ${ }^{18}$ Department of Entomology, Kansas State University, Manhattan, KS 66506, USA.

${ }^{19}$ Department of Biology, Divison of Developmental Biology, Friedrich-Alexander-University of Erlangen-Nürnberg, Staudtstr. 5, 91058 Erlangen, Germany. ${ }^{20}$ Department of Computational Molecular Biology, Max-Planck-Institute for Molecular Genetics, Ihnenstraße 63-73, 14195 Berlin, Germany. ${ }^{21}$ Department of Biology, Division of Developmental Biology, Friedrich-Alexander-University of Erlangen-Nürnberg, Staudtstr. 5, 91058 Erlangen, Germany. ${ }^{22}$ Oxford Brookes University, Centre for Functional Genomics, Gipsy Lane, Oxford OX3 OBP, UK. ${ }^{23}$ Department of Anatomy and Cell Biology, Wayne State University, Detroit, MI 48202, USA. ${ }^{24}$ Baylor College of Medicine, Houston, Texas, USA. ${ }^{25}$ Institute of Crop Science and Resource Conservation (INRES-Phytomedicine), Rheinische Friedrich-Wilhelms-University of Bonn, Bonn, Germany. ${ }^{26}$ Institute for Insect Biotechnology, Justus-Liebig University of Giessen, Heinrich-Buff-Ring 26-32, 35392 Giessen, Germany. ${ }^{27}$ Georg-August-Universität Göttingen, Göttingen, Germany.

Received: 30 August 2019 Accepted: 12 December 2019 Published online: 14 January 2020

\section{References}

1. Brown SJ, Shippy TD, Miller S, Bolognesi R, Beeman RW, Lorenzen MD, et al. The red flour beetle, Tribolium castaneum (Coleoptera): a model for studies of development and pest biology. Cold Spring Harb Protoc. 2009;2009:pdb. emo126.

2. Klingler M. Tribolium Curr Biol. 2004;14:R639-40.

3. Schröder R, Beermann A, Wittkopp N, Lutz R. From development to biodiversity--Tribolium castaneum, an insect model organism for short germband development. Dev Genes Evol. 2008;218:119-26.

4. Panfilio KA. Extraembryonic development in insects and the acrobatics of blastokinesis. Dev Biol. 2008:313:471-91.

5. Posnien N, Schinko JB, Kittelmann S, Bucher G. Genetics, development and composition of the insect head - a beetle's view. Arthropod Struct Dev. 2010;39:399-410.

6. Tautz D. Segmentation Dev Cell. 2004;7:301-12.

7. Davis GK, Patel NH. SHORT, LONG, AND BEYOND: Molecular and embryological approaches to insect segmentation. Annu Rev Entomol. 2002:47:669-99.

8. Snodgrass R. Insect metamorphosis: Smithsonian miscellaneous collections, V122, no. 9. Washington: Literary Licensing; 1954.

9. Bäumer D, Strohlein NM, Schoppmeier M. Opposing effects of notchsignaling in maintaining the proliferative state of follicle cells in the telotrophic ovary of the beetle Tribolium. Front Zool. 2012;9:15.
10. Tomoyasu Y, Wheeler SR, Denell RE. Ultrabithorax is required for membranous wing identity in the beetle Tribolium castaneum. Nature. 2005;433:643-7.

11. Hu Y, Schmitt-Engel C, Schwirz J, Stroehlein N, Richter T, Majumdar U, et al. A morphological novelty evolved by co-option of a reduced gene regulatory network and gene recruitment in a beetle. Proc R Soc B. 2018; 285:20181373.

12. Noh MY, Muthukrishnan S, Kramer KJ, Arakane Y. Cuticle formation and pigmentation in beetles. Curr Opin Insect Sci. 2016;17:1-9.

13. King B, Denholm B. Malpighian tubule development in the red flour beetle (Tribolium castaneum). Arthropod Struct Dev. 2014;43:605-13.

14. Li J, Lehmann S, Weißbecker B, Ojeda Naharros I, Schütz S, Joop G, et al. Odoriferous defensive stink gland Transcriptome to identify novel genes necessary for Quinone synthesis in the red flour beetle, Tribolium castaneum. PLoS Genet. 2013:9:e1003596.

15. Hunt T, Bergsten J, Levkanicova Z, Papadopoulou A, John OS, Wild R, et al. A comprehensive phylogeny of beetles reveals the evolutionary origins of a Superradiation. Science. 2007;318:1913-6.

16. Merzendorfer H, Kim HS, Chaudhari SS, Kumari M, Specht CA, Butcher S, et al. Genomic and proteomic studies on the effects of the insect growth regulator diflubenzuron in the model beetle species Tribolium castaneum. Insect Biochem Mol Biol. 2012;42:264-76.

17. Ulrich J, Dao VA, Majumdar U, Schmitt-Engel C, Schwirz J, Schultheis D, et al. Large scale RNAi screen in Tribolium reveals novel target genes for pest control and the proteasome as prime target. BMC Genomics. 2015;16. https://doi.org/10.1186/s12864-015-1880-y.

18. Berghammer AJ, Klingler M, Wimmer EA. A universal marker for transgenic insects. Nature. 1999:402:370-1.

19. Koniszewski NDB, Kollmann M, Bigham M, Farnworth M, He B, Büscher $M$, et al. The insect central complex as model for heterochronic brain development-background, concepts, and tools. Dev Genes Evol. 2016; 226:209-19.

20. Lorenzen MD, Berghammer AJ, Brown SJ, Denell RE, Klingler M, Beeman RW. piggyBac-mediated germline transformation in the beetle Tribolium castaneum. Insect Mol Biol. 2003;12:433-40.

21. Sarrazin AF, Peel AD, Averof M. A Segmentation Clock with Two-Segment Periodicity in Insects. Science. 2012;336(6079):338-41.

22. Schinko JB, Weber $M$, Viktorinova I, Kiupakis $A$, Averof $M$, Klingler $M$, et al. Functionality of the GAL4/UAS system in Tribolium requires the use of endogenous core promoters. BMC Dev Biol. 2010;10:53.

23. Schinko JB, Hillebrand K, Bucher G. Heat shock-mediated misexpression of genes in the beetle Tribolium castaneum. Dev Genes Evol. 2012;222:287-98.

24. Trauner J, Schinko J, Lorenzen M, Shippy T, Wimmer E, Beeman RW, et al. Large-scale insertional mutagenesis of a coleopteran stored grain pest, the red flour beetle Tribolium castaneum, identifies embryonic lethal mutations and enhancer traps. BMC Biol. 2009;7:73.

25. Beermann A, Jay DG, Beeman RW, Hulskamp M, Tautz D, Jürgens G. The short antennae gene of Tribolium is required for limb development and encodes the orthologue of the Drosophila distal-less protein. Development. 2001;128:287-97.

26. Brown SJ, Mahaffey JP, Lorenzen MD, Denell RE, Mahaffey JW. Using RNAi to investigate orthologous homeotic gene function during development of distantly related insects. Evol Dev. 1999;1:11-5.

27. Peel AD, Schanda J, Grossmann D, Ruge F, Oberhofer G, Gilles AF, et al. TCknirps plays different roles in the specification of antennal and mandibular parasegment boundaries and is regulated by a pair-rule gene in the beetle Tribolium castaneum. BMC Dev Biol. 2013;13:25.

28. Curtis CD, Brisson JA, DeCamillis MA, Shippy TD, Brown SJ, Denell RE. Molecular characterization of cephalothorax, the Tribolium ortholog of sex combs reduced. Genesis. 2001;30:12-20.

29. Bucher G, Scholten J, Klingler M. Parental RNAi in Tribolium (Coleoptera). Curr Biol. 2002;12:R85-6.

30. Tomoyasu Y, Denell RE. Larval RNAi in Tribolium (Coleoptera) for analyzing adult development. Dev Genes Evol. 2004;214:575-8.

31. Tomoyasu Y, Miller SC, Tomita S, Schoppmeier M, Grossmann D, Bucher G Exploring systemic RNA interference in insects: a genome-wide survey for RNAi genes in Tribolium. Genome Biol. 2008:9:R10.

32. Schmitt-Engel C, Schultheis D, Schwirz J, Ströhlein N, Troelenberg N, Majumdar $\mathrm{U}$, et al. The iBeetle large-scale RNAi screen reveals gene functions for insect development and physiology. Nat Commun. 2015;6:7822. 
33. Dönitz J, Schmitt-Engel C, Grossmann D, Gerischer L, Tech M, Schoppmeier $M$, et al. iBeetle-base: a database for RNAi phenotypes in the red flour beetle Tribolium castaneum. Nucleic Acids Res. 2015;43:D720-5.

34. Dönitz J, Gerischer L, Hahnke S, Pfeiffer S, Bucher G. Expanded and updated data and a query pipeline for iBeetle-base. Nucleic Acids Res. 2018;46:D831-5.

35. Gilles AF, Schinko JB, Averof M. Efficient CRISPR-mediated gene targeting and transgene replacement in the beetle Tribolium castaneum. Dev Camb Engl. 2015;142:2832-9.

36. Gilles AF, Schinko JB, Schacht MI, Enjolras C, Averof M. Clonal analysis by tunable CRISPR-mediated excision. bioRxiv. 2018:394221. https://doi.org/10 1101/394221.

37. Kim HS, Murphy T, Xia J, Caragea D, Park Y, Beeman RW, et al. BeetleBase in 2010: revisions to provide comprehensive genomic information for Tribolium castaneum. Nucleic Acids Res. 2010;38:D437-42.

38. Richards S, Gibbs RA, Weinstock GM, Brown SJ, Denell R, Beeman RW, et al. The genome of the model beetle and pest Tribolium castaneum. Nature. 2008:452:949-55.

39. Lorenzen MD. Genetic linkage maps of the red flour beetle, Tribolium castaneum, based on bacterial artificial chromosomes and expressed sequence tags. Genetics. 2005;170:741-7.

40. Boetzer M, Pirovano W. Toward almost closed genomes with GapFiller. Genome Biol. 2012;13:R56.

41. Shelton JM, Coleman MC, Herndon N, Lu N, Lam ET, Anantharaman T, et al. Tools and pipelines for BioNano data: molecule assembly pipeline and FASTA super scaffolding tool. BMC Genomics. 2015;16. https://doi.org/10. 1186/s12864-015-1911-8

42. Stanke M, Diekhans M, Baertsch R, Haussler D. Using native and syntenically mapped CDNA alignments to improve de novo gene finding. Bioinformatics. 2008;24:637-44.

43. Kent WJ. BLAT---the BLAST-like alignment tool. Genome Res. 2002;12: 656-64

44. Simão FA, Waterhouse RM, loannidis P, Kriventseva EV, Zdobnov EM BUSCO: assessing genome assembly and annotation completeness with single-copy orthologs. Bioinformatics. 2015;31:3210-2.

45. Gramates LS, Marygold SJ, dos Santos G, Urbano J-M, Antonazzo G, Matthews BB, et al. FlyBase at 25: looking to the future. Nucleic Acids Res. 2017:45:D663-71.

46. Elsik CG, Worley KC, Bennett AK, Beye M, Camara F, Childers CP, et al. Finding the missing honey bee genes: lessons learned from a genome upgrade. BMC Genomics. 2014;15:86.

47. Schwager EE, Sharma PP, Clarke T, Leite DJ, Wierschin T, Pechmann M, et al. The house spider genome reveals an ancient whole-genome duplication during arachnid evolution. BMC Biol. 2017;15:62.

48. O'Leary NA, Wright MW, Brister JR, Ciufo S, Haddad D, McVeigh R, et al. Reference sequence (RefSeq) database at NCBI: current status, taxonomic expansion, and functional annotation. Nucleic Acids Res. 2016;44:D733-45.

49. Raible F, Tessmar-Raible K, Osoegawa K, Wincker P, Jubin C, Balavoine G, et al. Vertebrate-type intron-rich genes in the marine annelid Platynereis dumerilii. Science. 2005:310:1325-6.

50. Otu HH, Sayood K. A new sequence distance measure for phylogenetic tree construction. Bioinformatics. 2003:19:2122-30.

51. Ulitsky I, Burstein D, Tuller T, Chor B. The average common substring approach to phylogenomic reconstruction. J Comput Biol J Comput Mol Cell Biol. 2006;13:336-50.

52. Ha M, Kim VN. Regulation of microRNA biogenesis. Nat Rev Mol Cell Biol. 2014;15:509-24.

53. Bartel DP. MicroRNAs: target recognition and regulatory functions. Cell. 2009;136:215-33.

54. Marco A, Hui JHL, Ronshaugen M, Griffiths-Jones S. Functional shifts in insect microRNA evolution. Genome Biol Evol. 2010. https:/doi.org/10.1093/gbe/evq053.

55. Ninova M, Ronshaugen M, Griffiths-Jones S. MicroRNA evolution, expression, and function during short germband development in Tribolium castaneum. Genome Res. 2016;26:85-96.

56. Enright AJ, John B, Gaul U, Tuschl T, Sander C, Marks DS. MicroRNA targets in Drosophila. Genome Biol. 2003;5:R1

57. Sun K, Jee D, de Navas LF, Duan H, Lai EC. Multiple in vivo biological processes are mediated by functionally redundant activities of Drosophila mir-279 and mir-996. PLoS Genet. 2015;11:e1005245.

58. Arakane Y, Muthukrishnan S, Beeman RW, Kanost MR, Kramer KJ. Laccase 2 is the phenoloxidase gene required for beetle cuticle tanning. Proc Natl Acad Sci U A. 2005;102:11337-42.
59. Lai Y-T, Deem KD, Borràs-Castells F, Sambrani N, Rudolf H, Suryamohan K, et al. Enhancer identification and activity evaluation in the red flour beetle, Tribolium castaneum. Development. 2018;145:dev160663.

60. Hinrichs AS. The UCSC genome browser database: update 2006. Nucleic Acids Res. 2006;34:D590-8.

61. Wu TD, Nacu S. Fast and SNP-tolerant detection of complex variants and splicing in short reads. Bioinformatics. 2010;26:873-81.

62. Dippel S, Oberhofer G, Kahnt J, Gerischer L, Opitz L, Schachtner J, et al. Tissue-specific transcriptomics, chromosomal localization, and phylogeny of chemosensory and odorant binding proteins from the red flour beetle Tribolium castaneum reveal subgroup specificities for olfaction or more general functions. BMC Genomics. 2014;15:1141.

63. Ashburner M, Ball CA, Blake JA, Botstein D, Butler H, Cherry JM, et al. Gene ontology: tool for the unification of biology. Gene Ontol Consortium Nat Genet. 2000;25:25-9.

64. Roberts A, Pachter L. Streaming fragment assignment for real-time analysis of sequencing experiments. Nat Methods. 2013;10:71-3.

65. Kriventseva EV, Tegenfeldt F, Petty TJ, Waterhouse RM, Simão FA, Pozdnyakov IA, et al. OrthoDB v8: update of the hierarchical catalog of orthologs and the underlying free software. Nucleic Acids Res. 2015; 43(Database issue):D250-6.

66. Stamatakis A. RAxML-VI-HPC: maximum likelihood-based phylogenetic analyses with thousands of taxa and mixed models. Bioinformatics. 2006;22: 2688-90.

67. Edgar RC. MUSCLE: a multiple sequence alignment method with reduced time and space complexity. BMC Bioinformatics. 2004:5:113.

68. Capella-Gutierrez S, Silla-Martinez JM, Gabaldon T. trimAl: a tool for automated alignment trimming in large-scale phylogenetic analyses. Bioinformatics. 2009;25:1972-3.

69. Zhang H, Gao S, Lercher MJ, Hu S, Chen W-H. EvolView, an online tool for visualizing, annotating and managing phylogenetic trees. Nucleic Acids Res. 2012;40:W569-72.

70. Kozomara A, Griffiths-Jones S. miRBase: annotating high confidence microRNAs using deep sequencing data. Nucleic Acids Res. 2014;42:D68-73.

71. St Pierre SE, Ponting L, Stefancsik R, McQuilton P, FlyBase Consortium. FlyBase 102--advanced approaches to interrogating FlyBase. Nucleic Acids Res. 2014:42(Database issue):D780-8.

72. Agarwal V, Bell GW, Nam J-W, Bartel DP. Predicting effective microRNA target sites in mammalian mRNAs. eLife. 2015;4:e05005.

\section{Publisher's Note}

Springer Nature remains neutral with regard to jurisdictional claims in published maps and institutional affiliations.

Ready to submit your research? Choose BMC and benefit from:

- fast, convenient online submission

- thorough peer review by experienced researchers in your field

- rapid publication on acceptance

- support for research data, including large and complex data types

- gold Open Access which fosters wider collaboration and increased citations

- maximum visibility for your research: over $100 \mathrm{M}$ website views per year

At $\mathrm{BMC}$, research is always in progress.

Learn more biomedcentral.com/submissions 